\title{
Development of a Telecommunication and Train Operation Simulator for Radio Communication Train Control
}

\author{
Yoko TAKEUCHI \\ Transport Operation Systems Laboratory, Signalling and Transport Information Technology Division \\ Kunihiro KAWASAKI \\ Signalling and Transport Information Technology Division \\ Yoichi SUGIYAMA \\ Train Control Systems Laboratory, Signalling and Transport Information Technology Division \\ When bringing radio communication train control systems into service, it is essential to \\ establish conditions that will allow stable radio communications, and take into account train \\ operating conditions. As such a radio telecommunications and train operations simulator to \\ forecast train operation was developed, that takes into account radio wave propagation and \\ telecommunications networks. In addition, using the simulator, a system design flow chart \\ was designed to ensure that certain basic criteria are met. This paper describes the simulator, \\ and the design flow chart, and presents the results from a case study carried out using the \\ simulator.
}

Keywords: radio communication train control system, simulation, train operation, radio link design, network design, deadlock

\section{Introduction}

Radio communication train control systems are systems which use radio communications in order to transmit train control information. Despite regional differences, this type of system is already in use in many countries around the world. Japan developed its own radio train control system called ATACS, which was brought into service in 2011 on the Senseki line and on the Saikyo line in 2017. JR West has announced that it will also be introducing the system on its Wakayama line. As such, this system is gradually being introduced across the Japanese railway network.

There are certain obvious premises when introducing radio train control systems: first, is the establishment of track-to-train communication, in addition to measures to guarantee the quality of data transmission. Traditionally, efforts were made to minimize the amount of trackside equipment required, which meant that only the minimum number of radio base stations were installed. However, the number of trains that a single base station can control is limited, so if there are not enough radio base stations on a busy line, not all planned transport services can be operated. In other words, when a radio link network is designed, operational needs must be taken into consideration.

Simulation tools to assist the work of radio-link network design have been developed by RTRI [1,2]. These simulators can evaluate a radio communications network taking into account complex radio propagation and interference environments. They were not designed however to simulate the variation in operating conditions of different operational time bands. Yet, as mentioned just before, it is important to ensure that if a radio train control system is introduced onto a busy line, it should not become a source of train delays.

RTRI has therefore been developing a "Telecommu- nications and Train Operation Simulator for Radio Communication Train Control" which not only simulates radio propagation and communication network transmission delays but can also forecast train operations [3, 4]. A design flow chart was also designed using this simulator [5].

Section 2 of this report gives an outline of the intended radio communication train control system, Section 2 describes the Telecommunications and Train Operation Simulator for Radio Communication Train Control, Section 4 introduces the communication network design flow chart, while Section 5 presents various case studies and Section 6 provides a number of conclusions and an outlook for future work.

\section{Overview summary of the radio communication train control}

Figure 1 shows the configuration of intended radio communication train control in this report. Each train set was equipped with radio equipment while base stations were installed along the track. When a predefined number of frames disappears in succession during the transmission process, which is called "frame loss," for a certain radio communication between the base station and the train, the system brings the train to an emergency stop for safety. Radio communication therefore must remain stable for stable train operation. Consequently, several frequency channels are made available and assigned to each base station as appropriate, in order to avoid wave interference.

The ground facility which receives authorizations for a train to proceed at an interlocking, collects train positions and calculates the stopping limit of each train, is called a "ground controller." Base stations are connected to ground controllers which in turn are connected to each other 
through the communication network. Data is sent and received at constant intervals between each piece of equipment to share the information needed to control traffic.

Each base station operates within a range along a line, which is called a "zone." The base station and the trains in its zone mutually send and receive train control information over a radio link. More specifically, the on-board device sends its position to the ground controller, and the ground controller sends the stopping limit to the on-board device. The on-board device then generates a speed curve using line data taken from the relevant database that already contains this information, and the speed limit which is sent from the ground controller, and limits train speed in accordance with the generated speed curve.

This report assumes use of the TDMA transmission system, whereby one base station can communicate with several trains at the same time. In the TDMA transmission system $^{\mathrm{A}}$, one frequency wave is split in time into regular "timeslots," each allocated to one train. As time runs, time slots switch between trains allowing them to communicate with the trains in rotation.

As each train moves along the line, it switches the base station used for communication. The crossover point, as a train moves between two base stations, is called the "handover position." This point marks a new starting position in preparation of the switch to the next base station, which is called "handover preparation position." When a train reaches handover preparation position and the base station detects it, the next base station prepares a time slot which will be assigned to that train in advance. At the next handover position, the system then switches base station, allowing smooth train operation control.

Stable train operation therefore requires sufficient allocation of radio resources, such as frequency channels and time slots.

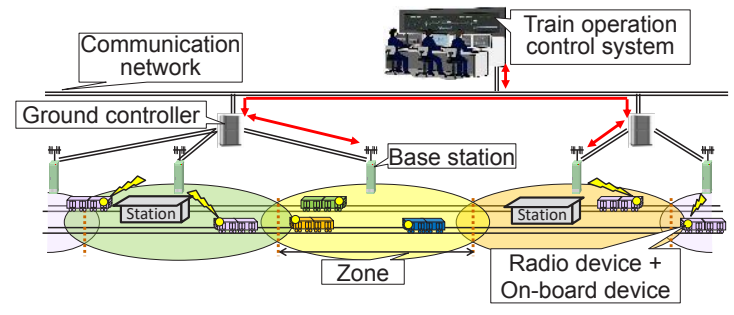

Fig. 1 Configuration of intended radio communication train control

\section{Telecommunications and Train Operation Simula- tor for Radio Communication Train Control}

When radio communication train control is used on a line with dense traffic, the radio link design must be designed to ensure that it does not become the cause of train delays. RTRI therefore developed a "Telecommunications and Train Operation Simulator for Radio Communication Train Control" which forecasts the performance of communication networks including radio and train operation. This

\footnotetext{
A It is the access method which multiple users or applications access the system at a regular intervals in order to mutual use of one frequency.
}

simulator can evaluate train operations, quality of radio communication and quantitatively forecast transmission delay for a potential radio communication train control system.

The Telecommunications and Train Operation Simulator for Radio Communication Train Control is coupled with 3 simulators: "RADTRACE" [1] to simulate radio communication, "TCNET" [2] to simulate communication network performance and "MATRIIX" [6] to simulate train operation. Figure 2 shows the configuration and inputoutput data of this simulator.

\subsection{Basic calculation procedures}

TCNET controls the time of the simulation. The system activates RADTRACE and MATRIIX successively to carry out small calculations at short time intervals. The bullet points (1) to (6) below correspond to steps (1) to (6) in Fig. 2.

(1) RADTRACE which simulates radio communication and calculates whether frame loss occurs or not.

(2) TCNET assigns time slots to trains. It calculates the round trip time ${ }^{\mathrm{B}}$ of communication networks.

(3) TCNET proceeds the simulation in time by a small calculation time margin.

(4) TCNET sends information about the current simulation time, time slot assignments and presence or absence of the need for an emergency stop caused by frame loss and the recovery of radio communication between the base station and the train that was stopped in an emergency to MATRIIX.

(5) MATRIIX calculates train operations up to the current simulation time using information from TCNET.

(6) MATRIIX sends information about each trains position, direction and velocity to TCNET. Procedure repeats from (1).

\subsection{Procedure for updating time slot assignment in- formation}

MATRIIX receives time slots assignment information for all trains for each calculation time unit. Updating time slot assignment information of a certain train indicates that the next zone's time slot is already assigned to that train to allow it to proceed into the next zone. MATRIIX then calculates the stopping limit and updates the speed curve.

\subsection{Procedure in case of successive frame loss}

When the predefined number of successive frame losses occurs during radio communication between the base station and a certain train, the system brings the train to an emergency stop for safety. RADTRACE calculates frame losses and TCNET determines the need or not for an emergency stop due to frame loss, and the recovery of radio communication between the base station and the train subject

в The time between the start of train control information transmission and reception of train response. 
to the emergency stop. TCNET sends this the outcome of this decision to MATRIIX for each calculation time unit. When an emergency stop is needed, MATRIIX triggers the emergency brake at the notified time. When the radio communication between the base station and the emergency stopping train recovers, MATRIIX allows the train to resume operation from the emergency stop position.

\subsection{Function of simple simulation for radio commu- nication}

RADTRACE calculations take time because they simulate radio propagation paths precisely. This simulator assumes that constant frame loss rate (FLR) and transmission delays do not affect train operations to allow simple radio communication simulation. Using these functions, a part of calculation can be skipped and the simulator can estimate train operation at high-speed.

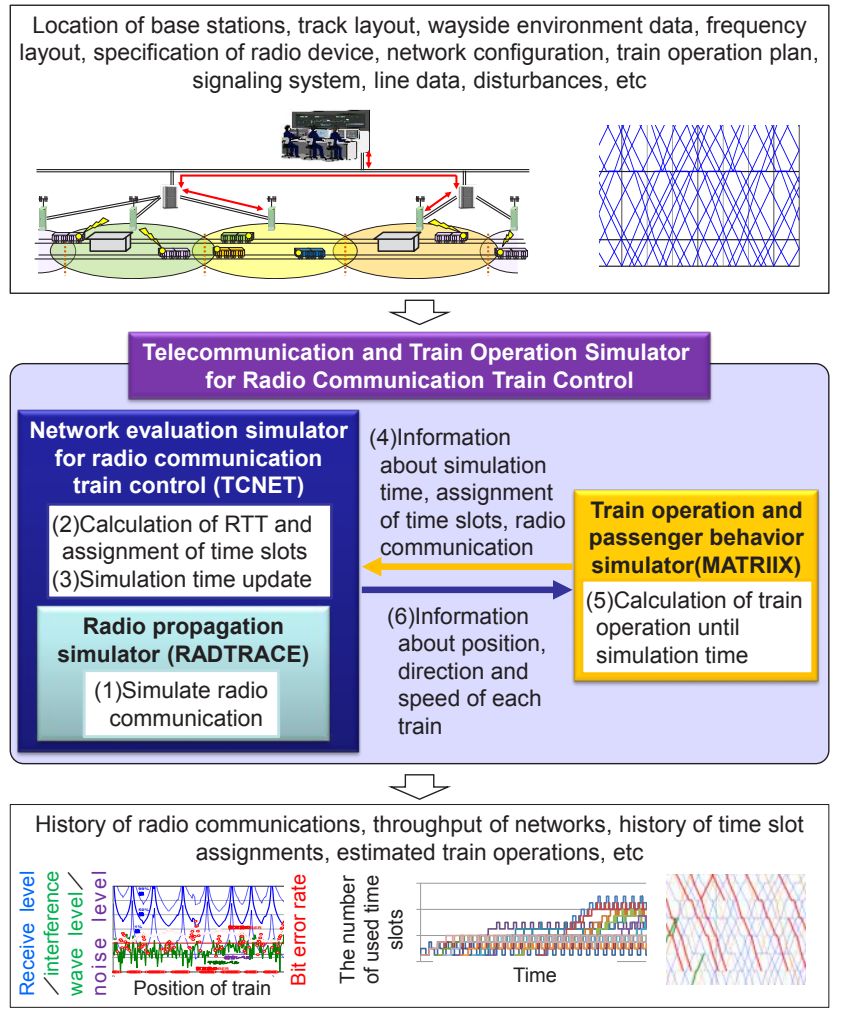

Fig. 2 Configuration and input-output data from Telecommunication and Train Operation Simulator for Radio Communication Train Control [5]

\section{Communication network design flow}

As described in Section 2, the intended radio communication train control described in this report must consider the constraints in Table1 in order to enable control using TDMA. In Table 1, the stability of radio communications, transmission delays on the communication network and the maximum number of controllable trains can be evaluated using the Telecommunications and Train Operation Simulator for Radio Communication Train Control. In addition, evaluating the probability of deadlock due to time slot assignment failure, is also important.
Therefore, a communications network design flow chart was designed, taking into account the constraints in Table 1 (Fig. 3). Firstly, railway companies set the thresholds for the five indices defining stable system operation. Depending on the characteristics of the line in question, not all five indices need to be fixed. Secondly, the minimum number of base stations is set as an initial solution and the flow chart in Fig. 3 is iterated until indices $I$ to $V$ satisfy the thresholds. The evaluation order of indices $\mathrm{I}$ to $\mathrm{V}$ is based on the difficulties of modifying the radio link design and the severity of conditions. Therefore, the radio link design can be adjusted to satisfy the requirements and the minimum number of base stations.

Indices $\mathrm{I}$ and $\mathrm{V}$ are explained in more detail in references [1] and [2], respectively. Index II is described in section 4.1. Indices III and IV are used by Telecommunications and Train Operation Simulator for Radio Communication Train Control. Section 5 describes a case study illustrating application of this design flow.

\subsection{Detection of deadlock probability}

The radio communication train control system needs to assign time slots to trains in order to run. In order to move from one zone to the next, trains need to be able to switch to the next base station to continue communications through a 'handover.' However, if all the time slots in the next base station are occupied by other trains, handover cannot occur and the train cannot proceed into the next zone. When time slots cannot be assigned, trains end up blocking a route. In such a situation, the system must release all time slots and re-assign them or change the train order. In this paper, this type of scenario is called a "deadlock."

Deadlocks have a huge impact on train operations, so it is crucial to avoid them. Based on the premise that the order of trains is not changed, this paper proposes a method for detecting potential deadlock zones using block sections, the maximum number of trains and route information.

Table 1 Constraints of radio communication train control [5]

\begin{tabular}{|l|l|}
\hline \multicolumn{1}{|c|}{ Constraints } & \multicolumn{1}{c|}{ Description } \\
\hline \multirow{2}{*}{$\begin{array}{l}\text { Stability of radio } \\
\text { communications }\end{array}$} & $\begin{array}{l}\text { If the distance between base stations is } \\
\text { too large, radio waves fail to reach } \\
\text { their destination. } \\
\text { If the distance between base stations is } \\
\text { too small, radio interference occurs. }\end{array}$ \\
\cline { 2 - 2 } & $\begin{array}{l}\text { When the predefined number of } \\
\text { successive frame losses occurs during } \\
\text { radio communication between the base } \\
\text { station and a certain train, the system } \\
\text { brings the train to an emergency stop. }\end{array}$ \\
\hline $\begin{array}{l}\text { Communication } \\
\text { network } \\
\text { transmission } \\
\text { delays }\end{array}$ & $\begin{array}{l}\text { If significant transmission delays } \\
\text { occur, the system may determine that } \\
\text { the critical number of frame losses has } \\
\text { been reached even though it has not. }\end{array}$ \\
\hline $\begin{array}{l}\text { Limit on number } \\
\text { of trains that can } \\
\text { be controlled }\end{array}$ & $\begin{array}{l}\text { Fixed maximum number of trains that } \\
\text { can be controlled at the same time by } \\
\text { one base station. }\end{array}$ \\
\hline Deadlock & $\begin{array}{l}\text { Deadlock which may occur due to time } \\
\text { slot assignment failure. }\end{array}$ \\
\hline
\end{tabular}




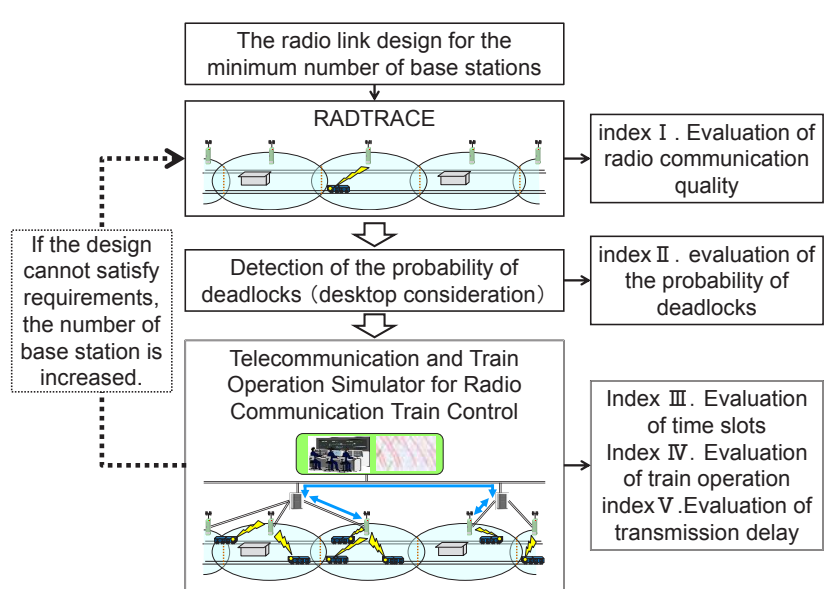

Fig. 3 Radio link design flow chart

\subsubsection{Deadlock detection without crossover}

Figure 4 shows the example of a deadlock. The time slots in zone 1 and zone 2 are occupied and train 1 and train 2 which are the first trains in each zone cannot proceed to the next zone, so a deadlock occurs. If only trains going in the same direction can occupy time slots in adjacent zones, deadlocks can occur.

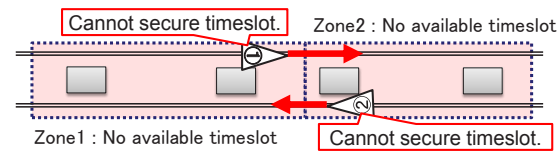

Fig. 4 Example of deadlock without crossover

\subsubsection{Detection of deadlock with crossover}

Figure 5 shows the example of the deadlock which is caused by the way the order of trains is controlled on crossing lines. Train 1 cannot proceed because there is no available time slot in zone 2 . To free a time slot in zone 2 , the train in zone 2 needs to proceed to zone 1 or zone 3 or another lines. However, train 3 cannot proceed because there are no available time slots in zone 3 . The running order of trains 1 and 4 is fixed in the train diagram, so, as train 1 precedes train 4 , train 4 is blocked. Figure 5 illustrates a deadlock where all trains are blocked unless they are reassigned time-slots or their running order is changed.

This type of deadlock occurs when there are no available time slots in sections bordered by two crossovers. Therefore, if the number of time slots in zones including a thick-frame part of Fig. 6 is more than the maximum num-

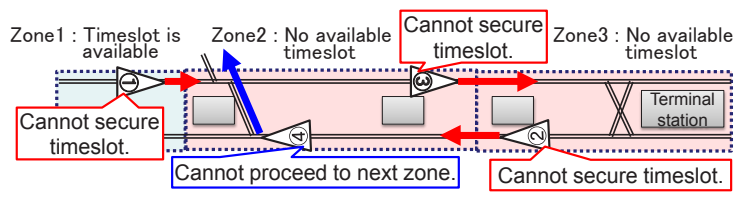

Fig. 5 Example of deadlock with crossover

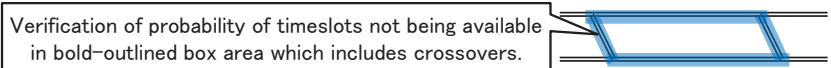

Fig. 6 Sections that need to be checked for deadlocks (box with bold-outline) ber of trains, there is no probability of the deadlock occurrence like Fig. 5.

\section{Case studies}

Two cases are presented using the flow chart in Fig.3.

\subsection{Example of the radio link design for high fre- quency train operation}

The first case study was on a real section of railway line with 29 stations and about $35 \mathrm{~km}$ long, with a virtual high frequency of trains with a headway of $1.5 \mathrm{~min}$. The railway company set thresholds only for indices I and III in Fig. 3, as shown in Table 2.

Firstly, the minimum number of base stations to satisfy index I was 13 . Secondly, a simulation using the Telecommunications and Train Operation Simulator for Radio Communication Train Control was used to confirm index III, which detected that time slots would run out. The number of base stations was therefore increased near that point and the simulation was run again. The result of the second simulation confirmed that there would be no shortage of time slots. Table 2 shows the evaluation results.

In this case study, the number of base stations installed to satisfy train operating conditions (14 base stations) exceeded the number required to satisfy radio communication quality (13 base stations). By applying the flow chart in Fig. 3, 14 was obtained as the minimum number of base stations that would be needed to satisfy both radio communications and transport service quality.

Table 2 Thresholds and evaluation results (Underlines represent the indices which cannot satisfy threshold)

\begin{tabular}{|c|c|c|c|}
\hline & & Index I & Index III \\
\hline \multicolumn{2}{|c|}{ Threshold } & $\begin{array}{c}\text { FLR }< \\
1.0 \times 10^{-3}\end{array}$ & $\begin{array}{l}\text { Shortage of timeslots } \\
\text { should not occur. }\end{array}$ \\
\hline \multirow{2}{*}{$\begin{array}{l}\text { Evaluation } \\
\text { results }\end{array}$} & $\begin{array}{l}13 \text { base } \\
\text { stations }\end{array}$ & $\begin{array}{c}\text { FLR }< \\
1.0 \times 10^{-3} \\
\end{array}$ & $\begin{array}{l}\text { Shortage of timeslots } \\
\text { occurs. }\end{array}$ \\
\hline & $\begin{array}{l}14 \text { base } \\
\text { stations }\end{array}$ & $\begin{array}{c}\text { FLR }< \\
1.0 \times 10^{-3}\end{array}$ & $\begin{array}{l}\text { Shortage of timeslots } \\
\text { does not occur. }\end{array}$ \\
\hline
\end{tabular}

\subsection{Example of radio link design considering train delay and equipment failure}

The second case study was on a real section of railway line with 19 stations and about $22 \mathrm{~km}$ long, and the railway company set thresholds for indices I, II, IV and V of Fig. 3. Thresholds are shown in Table 5 and 6. Figure 7 shows a part of train diagram.

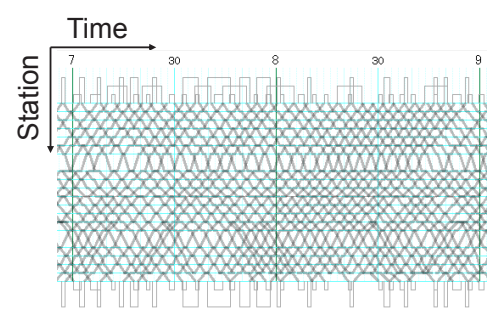

Fig. 7 Part of a train diagram [5] 


\subsubsection{Index I : Evaluation of radio communication quality}

Using the method from reference[1], a radio communication quality evaluation was carried out considering radio wave propagation, noise and wave interference. First, 8 base stations were installed, and then the height and position of the antenna and assignment of frequency channels were adjusted. Under these conditions, the FLR was less than $1.5 \times 10^{-3}$ so this design did not satisfy the threshold in Table 5. Second, the number of base stations was increased to nine, giving an FLR of less than $5.0 \times 10^{-2}$ for extension handovers ${ }^{\mathrm{C}}$ so this design did not satisfy the threshold again. Finally, 10 base stations were installed and other parameters were adjusted, giving a FLR of less than $1.0 \times 10^{-3}$ which meant that this design satisfied the threshold in Table 5 for handover.

\subsubsection{Index II : Evaluation of the probability of dead- locks}

To detect deadlock potential in a section without a crossover as described in 4.1.1, and whether there were sections where only trains moving in the same direction could occupy time slots in adjacent zones, the number of potential zones was verified. As a result, there were two locations where deadlocks could occur in case of concentrated traffic.

To detect potential deadlocks sections with a crossover as described in 4.1.2, sections bordered by two crossovers were identified (Fig. 8). Figure 8 shows that the number of trains needed to occupy all time slots in zone 2 to zone 4 including both crossovers was $12 \times 3=36$, however the previously established premise was a maximum of 25 trains. No deadlock was therefore detected in the section with two crossovers, so this design satisfied the threshold in Table 5.

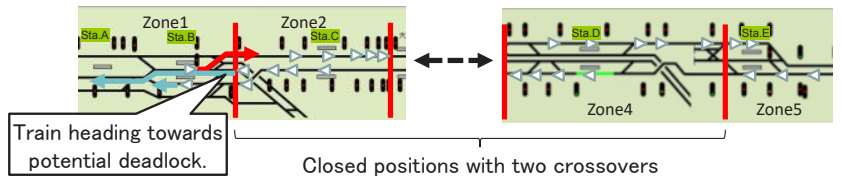

Fig. 8 Track layout from zone 2 to zone 4 [5]

\subsubsection{Index IV : Evaluation of train operation}

An evaluation was made to determine the possibility of delays due to the radio communications network not only during ordinary operation but also in case of localized heavy traffic. To simulate localized heavy traffic situations, four scenarios were set in Table 3 and 4, assuming absence or presence of the traffic disruption / equipment failure and the Telecommunication and Train Operation Simulator for Radio Communication Train Control was used to calculate the various iterations. The results showed that there were no train delays caused by the radio communication network, confirming that this design satisfied the threshold in Table 6

C When a certain radio equipment fails, adjacent zones is extended and the system is reset zones and handover positions as no such base station
Table 3 Train operation evaluation scenarios of train operation disruption [5]

\begin{tabular}{|l|l|}
\hline \multicolumn{1}{|c|}{ Situation } & \multicolumn{1}{c|}{ Consequence } \\
\hline $\begin{array}{l}\text { Disruption forcing trains } \\
\text { to concentrate in a certain } \\
\text { zone during morning rush } \\
\text { hour. }\end{array}$ & $\begin{array}{l}\text { Trains are rescheduled: five } \\
\text { minutes following disruption } \\
\text { all trains are suspended at } \\
\text { the nearest station. }\end{array}$ \\
\hline
\end{tabular}

Table 4 Train operation evaluation scenarios of equipment failure [5]

\begin{tabular}{|l|l|}
\hline \multicolumn{1}{|c|}{ Situation } & \multicolumn{1}{|c|}{ Consequence } \\
\hline Base station & $\begin{array}{l}\text { Implementation of extended handover: } \\
\text { down for a } \\
\text { adjacent zones each cover half of the zone } \\
\text { affected by the equipment failure. }\end{array}$ \\
\hline
\end{tabular}

\subsubsection{Index V : Evaluation of transmission delay}

To evaluate transmission delay, delays in transmission over the communication network were simulated and the RTT calculated through the Telecommunication and Train Operation Simulator for Radio Communication Train Control. Figure 9 shows the communication network design. Device performance was set to the standard value, FLR was set to the worst case scenario $1.0 \times 10^{-3}$, and train operation was set to normal. As a result, RTT was from 0.4 to $1.2 \mathrm{sec}$ (Fig. 10) confirming that this design satisfied the threshold in Table 6.

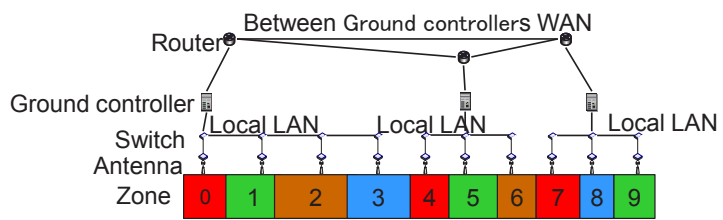

Fig. 9 Communication network design [5]

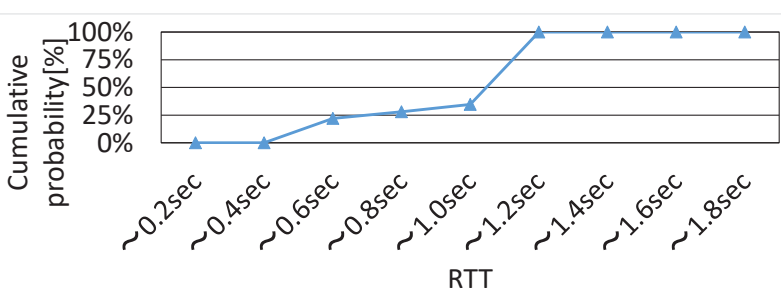

Fig. 10 Cumulative probability of RTT

\subsubsection{Summary of case study results}

Table 5 and 6 summarize the results from the case studies. Ten base stations satisfied the radio communication quality requirement and all the index requirements. This demonstrated that a radio link design needs at least ten base stations. 
Table 5 Thresholds and evaluation results (Underlined items represent the indices which do not satisfy the threshold)

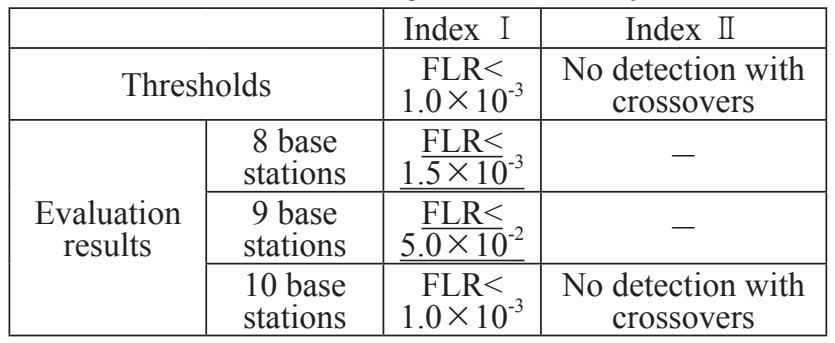

Table 6 Thresholds and evaluation results (Underlined items represent the indices which do not satisfy the threshold)

\begin{tabular}{|c|c|c|c|}
\hline \multicolumn{2}{|c|}{} & Index IV & Index V \\
\hline \multicolumn{2}{|c|}{ Thresholds } & No delay & RTT $<3.0[\mathrm{~s}]$ \\
\hline \multirow{4}{*}{$\begin{array}{c}\text { Evaluation } \\
\text { results }\end{array}$} & $\begin{array}{c}8 \text { base } \\
\text { stations }\end{array}$ & - & - \\
\cline { 2 - 4 } & $\begin{array}{c}9 \text { base } \\
\text { stations }\end{array}$ & - & - \\
\cline { 2 - 4 } & $\begin{array}{c}10 \text { base } \\
\text { stations }\end{array}$ & No delay & RTT $<1.2[\mathrm{~s}]$ \\
\hline
\end{tabular}

\section{Conclusions}

This paper describes the "Telecommunication and Train Operation Simulator for Radio Communication Train Control" which can simulate both radio communication networks and train operations. A method was also developed to detect deadlocks due to installation of a radio communication train control system. Based on this work, a radio link design flow chart was developed to produce designs that satisfy the various thresholds and set the minimum number of required base stations. Finally, the results were presented from a number of case studies applying these methods.

Although the simulator was developed for JRTC type radio communication train controls, designed and in use in Japan, it can be customized for CBTC which uses 2.45G band or $5 \mathrm{G}$ band. Future work will aim to develop a method to assist radio link design for installing radio communication train control systems using these results.

\section{Acknowledgement}

The authors would like to express their sincere gratitude to the East Japan Railway Company and the West Japan Railway Company.

\section{References}

[1] Kawasaki, K., Sugahara, H., Tateishi, K., and Hattori, T., "Implementation and Verification of a Digital Radio Communication System Simulator," IEEJ Transactions on Industry Applications, I.E.E. Japan, Vol. 135, No. 4, pp.420-425, 2015 (in Japanese).

[2] Sugahara, H., "Performance Evaluation System of Communication Network for Train Control System Using Radio Communication," Quarterly Report of RTRI, Vol.56, No.3, pp.187-193, 2015.

[3] Takeuchi, Y., Kawasaki, K., and Sugahara, H., "Coupled Simulation for Radio Train Control System Design," Joint Technical Meeting on "Linear Drives" and "Transportation and Electric Railway," I.E.E. Japan, LD-16-056/ TER-16-063, 2016 (in Japanese).

[4] Takeuchi, Y., and Kawasaki, K. "Evaluation Flow for Radio Train Control System Design by means of Simulation Systems," J-Rail2016, S4-1-4, pp.305-308, 2016 (in Japanese).

[5] Takeuchi, Y., Kawasaki, K., and Sugiyama, Y., and Sakaguchi, T., "Development of Telecommunication and Train Operation Simulator for Radio Communication Train Control And System Design Flow," Technical Meeting on "Transportation and Electric Railway," I.E.E. Japan, TER-18-059, 2018 (in Japanese).

[6] Takeuchi, Y., Sakaguchi, T., Kumazawa, K., Kunimatsu, T., and Sato, K., "A Train Operation Simulation System Based on a Detailed Model of Train Running Performance," RTRI REPORT, No.28, Vol.4, pp.41-46, 2014 (in Japanese).

\section{Authors}

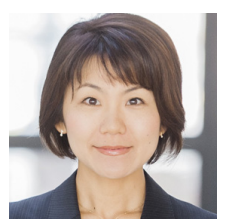

Yoko TAKEUCHI

Senior Researcher, Transport Operation Systems Laboratory, Signalling and Transport Information Technology Division

Research Areas: Train Operation Simulation, Energy Simulation and Control

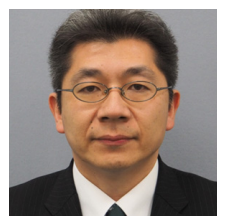

Kunihiro KAWASAKI

Director, Head of Signalling and Transport Information Technology Division Research Areas: Communication Network

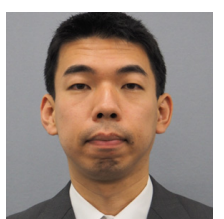

Yoichi SUGIYAMA

Assistant Senior Researcher, Train Control Systems Laboratory, Signalling and Transport Information Technology Division Research Areas: Radio Train Control System 